\title{
Status of ATLAS diamond Beam Condition Monitor
}

\author{
Andrej Gorišek $^{\text {a,1 }}$, V. Cindro a , I. Dolenc ${ }^{\text {a }}$, H. Kagan ${ }^{\text {b }}$, G. Kramberger ${ }^{\text {a }}$, \\ H. Frais-Kölbl c , E. Griesmayer ${ }^{\text {c }}$, B. Maček ${ }^{\text {a }}$, I. Mandić ${ }^{\text {a }}$, M. Mikuž ${ }^{\text {a }}$, M. Niegl ${ }^{\text {c }}$, \\ H. Pernegger ${ }^{\text {d }}$, S. Smith ${ }^{b}$, D. Tardif ${ }^{e}$, W. Trischuk ${ }^{\text {e }}$, P. Weilhammer ${ }^{\text {, }}$, \\ M. Zavrtanik ${ }^{\text {a }}$
}

\author{
${ }^{a}$ J. Stefan Institute, Ljubljana, Slovenia \\ ${ }^{b}$ Ohio State University, Columbus, USA \\ ${ }^{c}$ Univ. of Applied Science, Wiener Neustadt, Austria \\ ${ }^{d}$ CERN, Geneva, Switzerland \\ ${ }^{e}$ University of Toronto, Toronto, Canada
}

\begin{abstract}
Beam conditions and the potential detector damage resulting from their anomalies have pushed the LHC experiments to plan their own monitoring devices. The ATLAS Beam Conditions Monitor (BCM) consists of two sets of four detector modules. These are required to be radiation hard (50 Mrad and $10^{15}$ particles $/ \mathrm{cm}^{2}$ ). Each module includes two diamond pad sensors read out in parallel. The sets are located symmetrically around the interaction point at $\mathrm{z}= \pm 184 \mathrm{~cm}$ and $\mathrm{r}=55 \mathrm{~mm}$ (a pseudo-rapidity of about 4.2 ). Equipped with fast electronics ( $2 \mathrm{~ns}$ shaping time) these stations measure time-of-flight and pulse height to distinguish events resulting from beam anomalies from those normally occurring in proton-proton interactions. The BCM also provides a coarse measurement of bunch-by-bunch luminosities in ATLAS by counting in-time and outof-time collisions. Eleven detector modules have been fully tested and assembled. Tests performed range from full characterization of diamond sensors to full module tests with electron sources and pion test-beams. Test-beam results in the CERN PS show a module signal to noise of about 11:1 for minimum ionizing particles. The best eight modules have now been installed on the ATLAS Pixel support frame that was installed in ATLAS during the summer of 2007. The final BCM system will be described as well as results of system optimisation, quality assurance, electronics performance evaluation and irradiation tests.
\end{abstract}

Vertex 2007

16th International Workshop on Vertex detectors

September 23-28, 2007, Lake Placid, NY, USA

\footnotetext{
$1 \quad$ Speaker; Address: J. Stefan Institute, Jamova 39, 1000 Ljubljana; E-mail: andrej.gorisek@ijs.si
} 


\section{Introduction}

Potential detector damage resulting from abnormal beam conditions could damage ATLAS Inner Detector which encouraged the implementation of Beam Condition Monitor (BCM) [1]. The aim of ATLAS BCM is to monitor the beam conditions and luminosity very close to the ATLAS interaction point, inside the Inner Detector. It consists of 8 detector modules organized in two sets of four modules on each side of the interaction point. The pCVD diamond sensors of each of the BCM modules are located symmetrically around the interaction point at $z=184 \mathrm{~cm}$ and $r=55 \mathrm{~mm}$ and supported on the ATLAS Pixel carbon fibre structure which also serves for the support of BCM supply and signal cables. Figure 1 shows the mechanical mounting and the inside of one of the modules with the diamond sensors and front end electronics visible.

The modules are required to be radiation hard since they will be installed close to interaction point and close to the beam-pipe in the place where expected radiation will reach doses of about $50 \mathrm{Mrad}$ and fluences of about $10^{15}$ particles $/ \mathrm{cm}^{2}$ in 10 years of operation of ATLAS at nominal luminosity. pCVD diamonds were chosen for their radiation hardness and fast signal response which allows to measure the beam conditions on the bunch by bunch basis. They were also shown to draw only tiny leakage currents allowing for no active cooling which is neither required for the amplifiers that can dissipate the heat by convection to the surrounding Pixel gas volume.

Each BCM module consists of two polycrystalline CVD (Chemical Vapour Deposition) diamond pad sensors mounted on top of each other and connected in parallel [2]. Signals created in the diamond sensors by charged particles are amplified in two stage amplifier build of off-the-shelf components [3] which were chosen for their satisfactory performance after the irradiation. Further away from the interaction point (in radiation less harsh environment) the precise timing information and amplitude is encoded into digital signals sent to the back end electronics based on sophisticated FPGA board. The principle of operation is shown on Figure 2 where interactions at interaction point will cause the appearance of signals in modules on both sides simultaneously ("in time events") and the background events which will cause time difference between the recorded signals on the two sides of $12.5 \mathrm{~ns}$ ("out of time events"), about $1 / 2$ of the bunch to bunch time spacing due to adequately chosen BCM module locations.

In addition to beam condition monitoring the $\mathrm{BCM}$ will provide valuable complementary luminosity monitoring information which could be used for example for correcting the trigger for bunch to bunch luminosity variations. During the LHC early commissioning when the conditions will not yet be stable and the Inner Detector will most likely be switched off, the BCM might be the first detector system to report proton-proton collisions inside ATLAS spectrometer (maybe even proton-beam-gas collisions as early as when the first single circulating LHC beam is commissioned). 

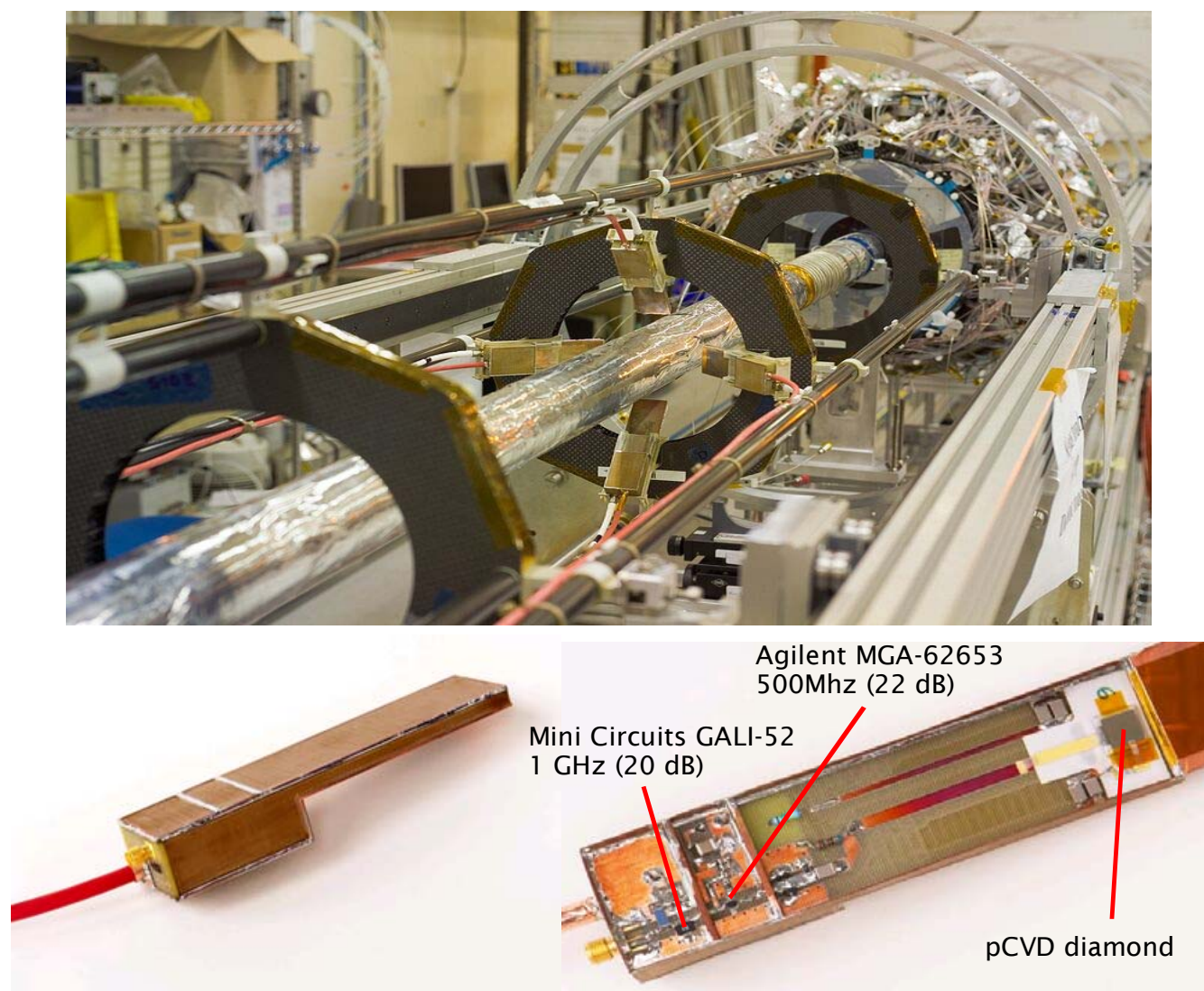

Figure 1: Four ATLAS BCM modules installed on the side-C of the ATLAS Pixel system support frame (upper picture). The beam-pipe going through the support structure as well as Pixel detector in the background can also be seen. The lower two pictures show BCM module soldered close (lower left picture) and BCM module prior to installation of the covers where pCVD diamond sensors and two stage amplifier is visible (lower right picture).

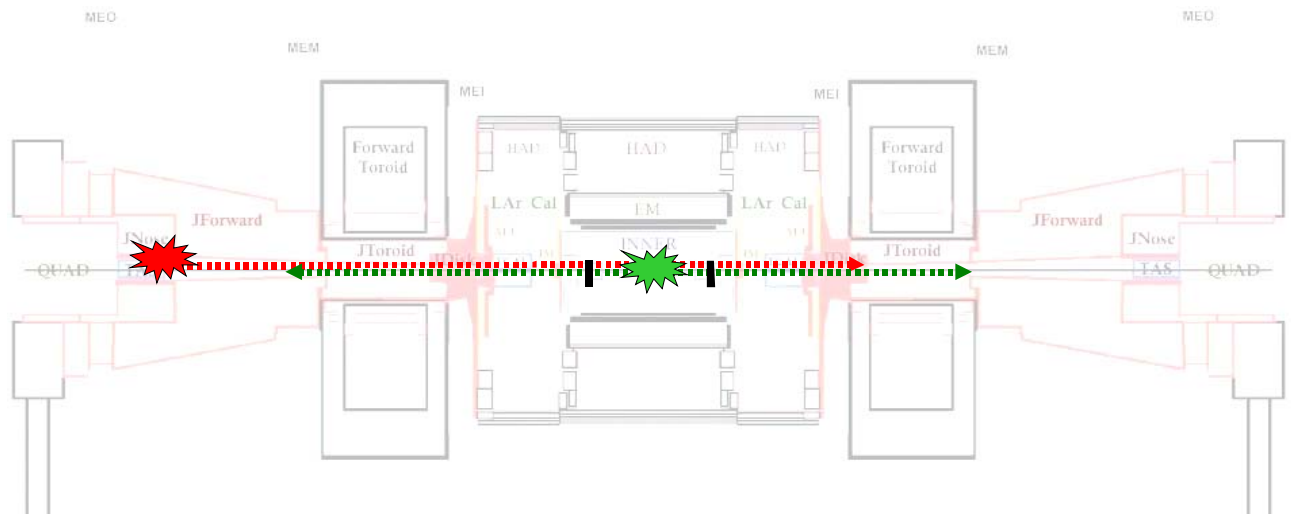

Figure 2: Principle of operation of Beam Condition Monitor. In case of the normal proton-proton interaction at interaction point (green) secondary particles would reach both sides of BCM system simultaneously. In the case of anomalous event such as proton hitting a collimator (red) one side of BCM system would detect secondary particles before the other. The $z= \pm 184 \mathrm{~cm}$ defines the time difference between the two sides to $\sim 12.5$ ns almost exactly $1 / 2$ of the time interval between two consecutive LHC bunches. 


\section{The BCM detector module}

The polycrystalline CVD diamond sensors of $1 \mathrm{~cm} \times 1 \mathrm{~cm}$ size and $500 \mu \mathrm{m}$ thickness were developed by a collaboration of RD42 [5] and Element Six Ltd. [6] (later named Diamond Detectors Ltd.). They were proven to be radiation hard and to produce very fast signals (rise time $<1 \mathrm{~ns}$, signal width $\sim 2 \mathrm{~ns}$ ). They are also attractive choice due to low leakage currents even after irradiation which does not exceed $1 \mathrm{nA} / \mathrm{cm}^{2}$ thus no active cooling is required to cool the sensors. Two pad sensors are assembled back to back on a $\mathrm{Al}_{2} \mathrm{O}_{5}$ ceramic baseboard which brings high voltage $( \pm 1000 \mathrm{~V})$ to the lower surface of the bottom diamond sensor and to the pad on the side which is used to connect the high voltage to the upper side of the top diamond sensor through several bond wires. The middle surfaces of the two sensors are conductively glued together with small pieces of ceramic distance holders and connected to the signal line on the ceramic baseboard through multiple bonds (see Figure 1). All parts are glued together with thermoplastic conductive adhesive pads (Staystik 571, a material proven to be radiation hard).

Ceramic module with two diamond sensors was assembled into the front end electronics box [3] based on commercially available current amplifiers: $500 \mathrm{MHz}$ Agilent MGA-62563 GaAs MMIC and $1 \mathrm{GHz}$ Mini Circuits Gali 52 HBT chips, each providing amplification of about $20 \mathrm{db}$. The first stage Agilent amplifier has an excellent noise performance with its noise figure below $1 \mathrm{db}$ in most of its frequency range.

To avoid electrical interference and pick-up each module was closed by soldering covers to each of the three compartments on the upper side (respectively containing two diamond sensors, first stage Agilent amplifier and second stage Gali amplifier, see Figure 1) and the compartment on the lower side containing the LV/HV power cable connector and temperature sensor.

\section{The ATLAS BCM system}

The ATLAS BCM will measure the arrival times of the signals and their time over threshold (TOT) for each of the 8 modules. These signals will be processed in real-time into rates, trends of rates for individual modules as well as for different logical combinations of signals taking into account also the timing within $25 \mathrm{~ns}$ ("in time" and "out of time" signals). The Figure 3 schematically shows the BCM system architecture. BCM system functionality can be summarized as follows:

- LHC Beam Abort. Two redundant signals indicating that beam conditions in ATLAS Inner Detector have reached the beam abort levels are sent to LHC through ATLAS "CIBU" system and will eventually cause that all LHC bunches being dumped in a controlled manner.

- ATLAS Detector Safety System. 4 signals are sent to ATLAS DSS, the hardware interlock system, which will allow for the ATLAS Inner Detector components to react in order to protect their hardware.

- ATLAS Detector Control System. In less severe cases and in addition to the hardware based DSS the warnings and alarms will be sent also through the ATLAS DCS. More sophisticated information and histograms will also be available through DCS system to ATLAS and LHC control. All recent information from BCM stored in circular buffer of the back end electronics will also be dumped through this channel. 
- ATLAS Level 1 trigger information. ATLAS BCM will provide also a 9-bit information for the ATLAS Level 1 trigger system, allowing for triggering on topologically interesting events. BCM can provide in real time bunch to bunch luminosity variation information - a valuable input to the trigger system.

- $\quad$ ATLAS DAQ stream. Digitized information of signal arrival times and their widths will be stored into special buffer in the ATLAS DAQ data stream. BCM data bits will be also recorded in the trigger data block.

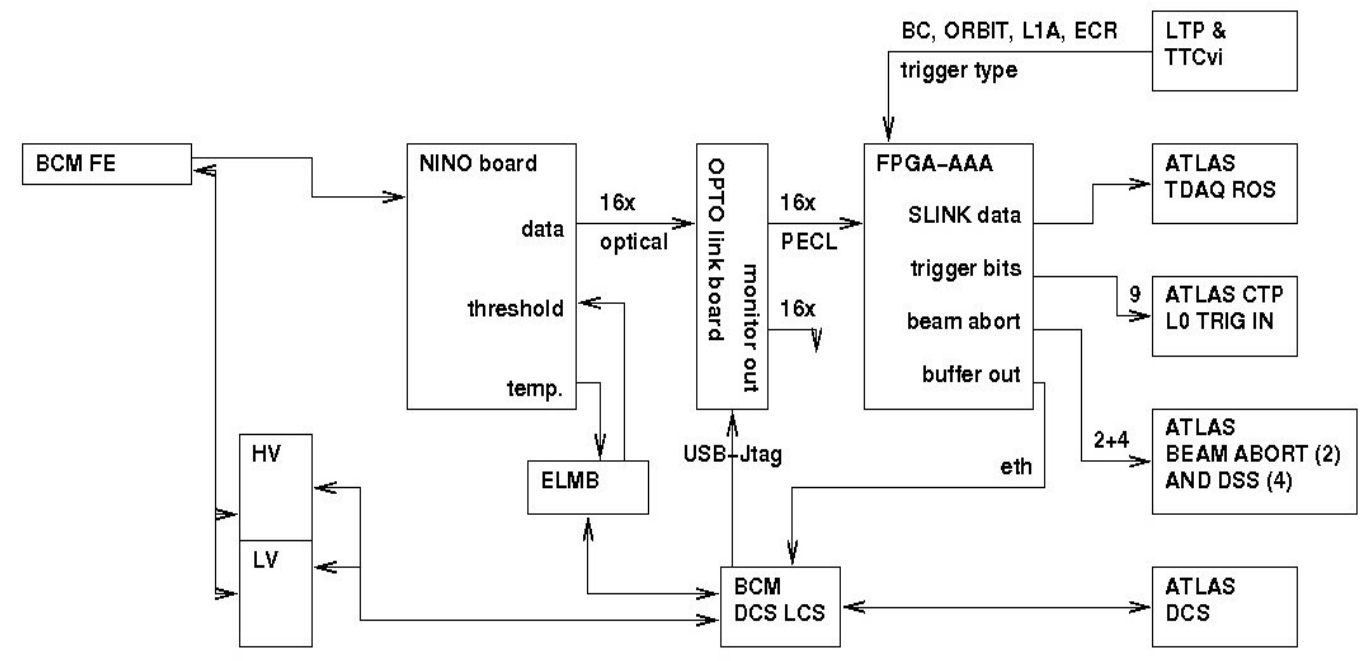

Figure 3: Symbolic BCM connectivity diagram.

Signals from BCM modules are taken through $14 \mathrm{~m}(2 \mathrm{~m}$ of Gore 41 in the inside and $12 \mathrm{~m}$ of Andrew HELIAX FSJI on the outside of the Pixel volume) of coaxial cables to a region with lower radiation where radiation tolerant electronics can be used. This electronics is based on NINO chip [4] designed by CERN-MIC for ALICE RPC detector for time of flight measurement. The NINO ASIC has 8 channels and features differential input amplifier (1 ns peaking time, $25 \mathrm{ps}$ jitter), discriminator and the time over threshold measurement. It is built in radiation tolerant $1 / 4 \mu \mathrm{m}$ IBM technology. The NINO electronic board first filters (low-pass fourth order filter with bandwidth of $\sim 300 \mathrm{MHz}$ ) and splits the signals from each of the BCM FE modules into two parts in ratio of approximately 1:11 to increase the dynamic range of the BCM system. These signals are then fed into two input channels of NINO chip. The ground of each BCM channel separately (BCM module, NINO electronics, HV and LV power supply channel) is kept electrically floating with connection only to the Pixel reference ground through a $1 \mathrm{k} \Omega$ resistor close to BCM modules. The TOT digital outputs of the NINO chip is converted into optical signals using radiation tolerant laser diodes (Mitsubishi FU-427SLD-FV1) and taken with $70 \mathrm{~m}$ optical fibres to the ATLAS counting room where they are received by photo diodes (Lightron LP3A4-SNC1) converted to PECL electric levels and fed into two Xilinx ML410 development boards based on Xilinx Vitrex-4 FPGA chip. Optical receiver board also provides monitoring NIM level outputs. Each Xilinx ML410 boards features 8 RocketIO serial input/output channels that will sample signals received at frequencies of $2.56 \mathrm{GHz}$. The onboard RAM memory banks act as ring buffers to store the BCM signal information for the time of the last several hundreds of LHC bunch crossings. This information will be used for the post- 
mortem analysis after a potential beam dump. The processed data from the Xilinx ML410 will be sent through ATLAS standard optical link ("S-LINK") to the ATLAS DAQ system, via Ethernet to the ATLAS DCS system and electrically to the ATLAS interlock (DSS) system.

\section{4. $\quad$ Results}

\subsection{Design optimisation}

The prototype modules and electronics as well as the final modules were tested with electrons from ${ }^{90} \mathrm{Sr}$ source and in numerous test beam measurement [7]. Earlier measurements were targeted to the detector module and front-end electronics design which was optimized to maximize the signal-to-noise figure (SNR). By using two diamonds on top of each other ("double decker" configuration) reading them in parallel we gain factor of 2 in signal while noise is increased only for $\sim 30 \%$. Inclining the modules at $45^{\circ}$ to the beam-pipe direction the signal for an average track in ATLAS (almost parallel to the beam-pipe) is increased by square root of 2 without any change in noise (the effective active area is decreased, though). The last two arguments are symbolically shown in Figure 4. Further it was shown that there is no increase in SNR when using thinner $350 \mu \mathrm{m}$ diamonds running them at unchanged voltage (increased field) instead of "standard" $500 \mu \mathrm{m}$. It was established that limiting the bandwidth of the signal by low-pass filter of about $200-300 \mathrm{MHz}$ optimizes SNR (see Figure 5) which is also indicated in Figure 6, which shows two different sets of measurements, first at full bandwidth of the $1 \mathrm{GHz}$ LeCroy oscilloscope and second at bandwidth limited to $200 \mathrm{MHz}$.
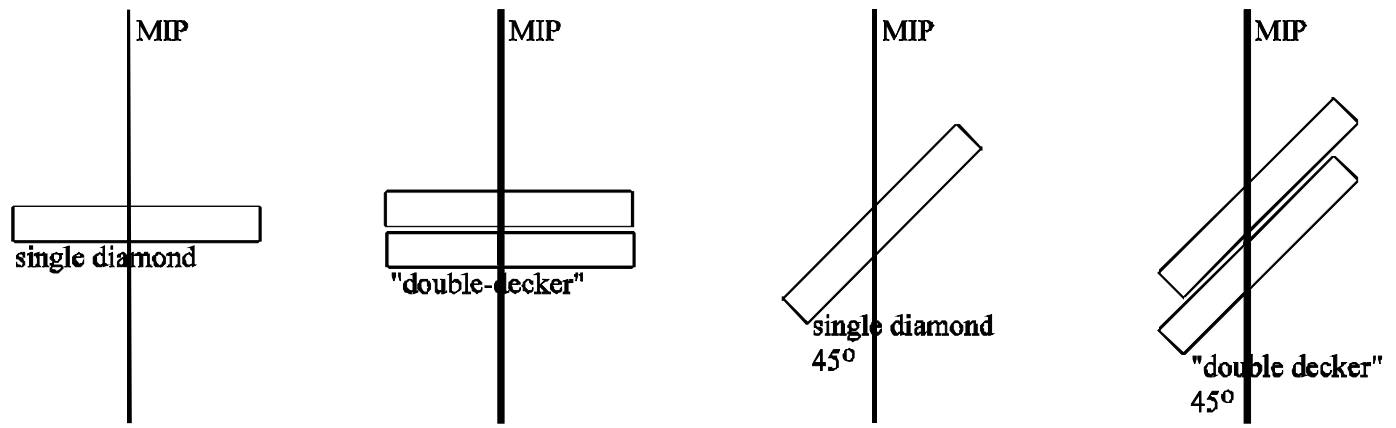

Figure 4: The design considerations. Final system has double sensor (“double decker") configuration at $45^{\circ}$ towards beam direction.
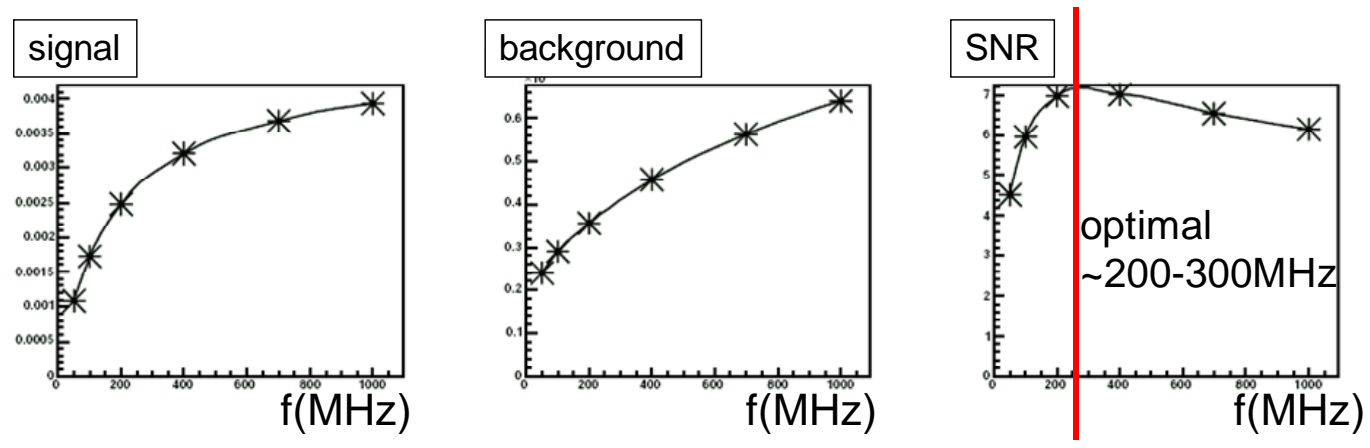
Figure 5: Simulated effect of low-pass RC filter to signal to noise figure shows an optimum frequency around 200-300 MHz.
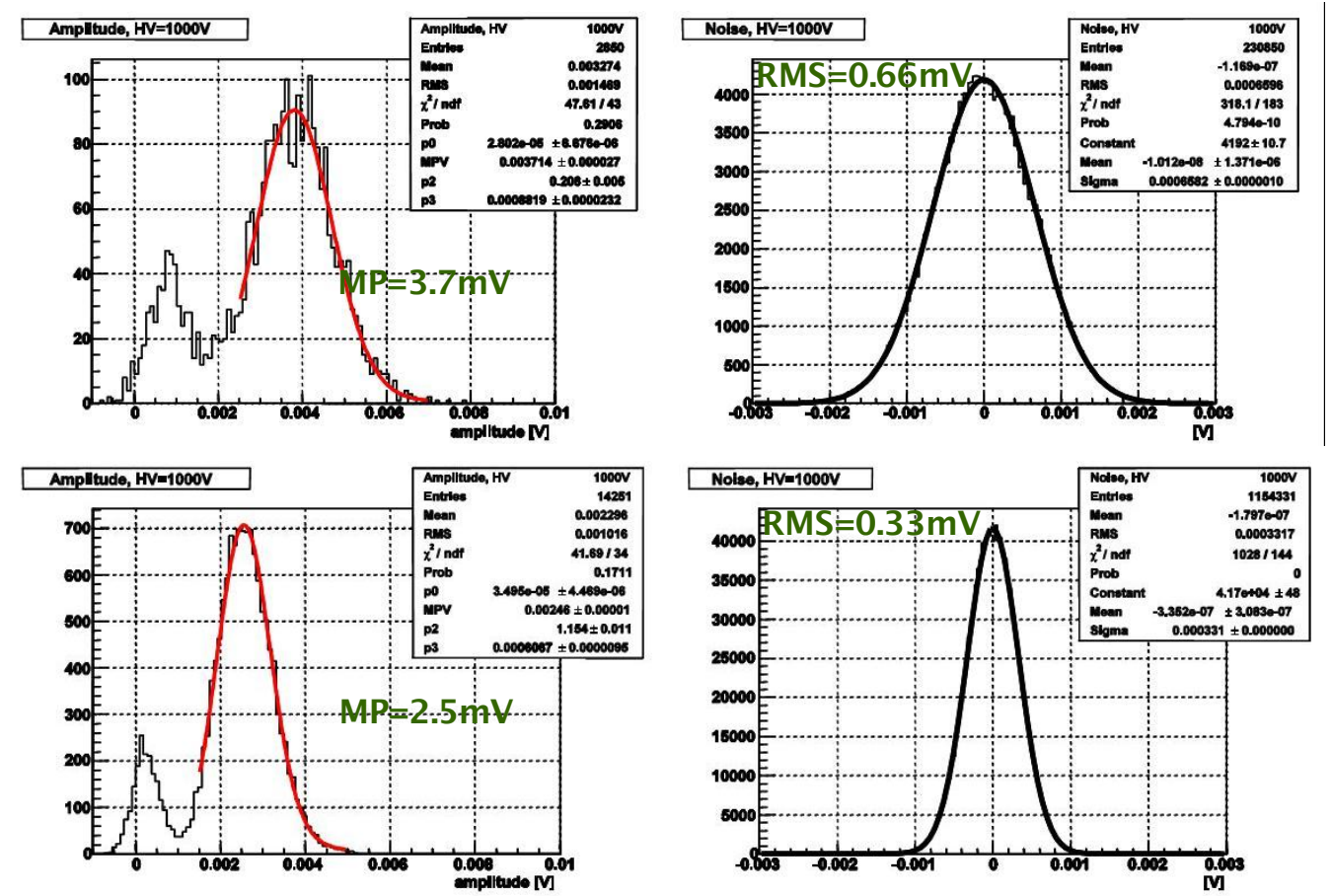

Figure 6: Limiting bandwidth to $200 \mathrm{MHz}$ (lower plots) decreases the signal for about 30\% compared to the full bandwidth (upper plots) while decreasing the noise by factor of 2 thus increasing the signal to noise figure for about $50 \%$.

\subsection{Quality assurance and installation of the BCM FE modules}

In 2006 a rigorous QA procedure was applied to all final BCM modules which included initial performance evaluation with electrons in ${ }^{90} \mathrm{Sr}$ setup. After the boxes were sealed closed with covers soldered on the top of each of the four compartments they went through the evaluation procedure again. After that they were kept under high voltage bias and with the front end amplifiers powered at $80^{\circ}$ for $12 \mathrm{~h}$ and cycled thermally from $-25^{\circ}$ to $40^{\circ}$ (more than the temperature variation expected in the installed system) for 10 times, after which their performance were re-evaluated. All modules were tested also in test beam at CERN PS and SPS in 2006 prior to installation. Four BCM modules at a time were installed between two sets of silicon strip detectors forming a beam telescope (see Figure 7). From the accurate telescope information on the incidence position of the pion was obtained $(\sim 10 \mu \mathrm{m}$ resolution in perpendicular direction to the beam). No surface variation of MIP detection efficiency was seen. The best eight modules were selected and installed on the ATLAS Pixel support structure. Table 1 shows the summary of performance figures obtained in the electron ${ }^{90} \mathrm{Sr}$ on-the-bench setup for the eight modules selected. Their signal to noise figures average at $7.3 \pm 0.6$ for the perpendicular incidence of electrons in ${ }^{90} \mathrm{Sr}$ setup which is also compatible with signal to noise figures obtained in the test beams of about $11: 1$ at $45^{\circ}$ incidence. 


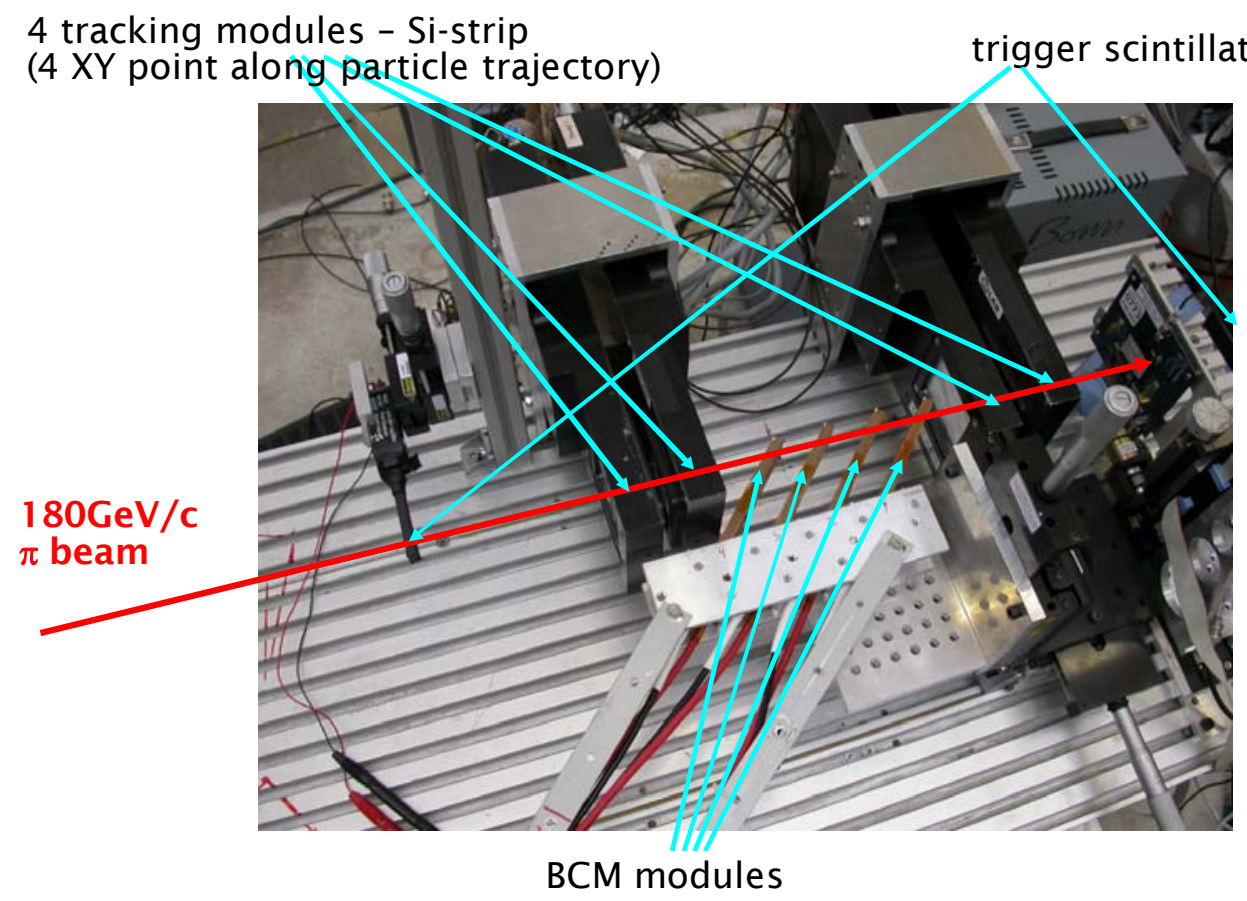

Figure 7: CERN PS/SPS beam test setup with 4 tracking detectors installed for reconstructing position information of traversing tracks.

\begin{tabular}{|l|c|c|c|c|c|c|c|c|}
\hline MODULE & $\mathbf{4 1 0}$ & $\mathbf{4 1 3}$ & $\mathbf{4 2 0}$ & $\mathbf{4 2 2}$ & $\mathbf{4 0 4}$ & $\mathbf{4 0 5}$ & $\mathbf{4 0 8}$ & $\mathbf{4 2 4}$ \\
\hline $\begin{array}{l}\text { preferred } \\
\text { polarity }\end{array}$ & $+1000 \mathrm{~V}$ & $+1000 \mathrm{~V}$ & $-1000 \mathrm{~V}$ & $-1000 \mathrm{~V}$ & $+1000 \mathrm{~V}$ & $+1000 \mathrm{~V}$ & $-1000 \mathrm{~V}$ & $-1000 \mathrm{~V}$ \\
\hline MPV [mV] & 2.77 & 2.15 & 2.67 & 2.40 & 2.43 & 2.30 & 2.21 & 2.70 \\
\hline SNR & $\mathbf{7 . 8}$ & $\mathbf{7 . 0}$ & $\mathbf{7 . 8}$ & $\mathbf{7 . 3}$ & $\mathbf{6 . 5}$ & $\mathbf{7 . 0}$ & $\mathbf{7 . 0}$ & $\mathbf{8 . 2}$ \\
\hline
\end{tabular}

Table 1: Summary of modules selected for installation. Table shows the preferred polarity of the module (at which signal is larger and leakage current smaller then at the opposite), most probable values of signal recorded at this polarity in ${ }^{90} \mathrm{Sr}$ setup and corresponding signal to noise figures. 


\subsection{Irradiations}

The front end amplifiers were tested for radiation hardness. The crucial first stage Agilent amplifier was shown to lose about $20 \%$ of its original amplification with no significance increase in noise.

The best of the three spare modules (F401) was chosen to go through multi step irradiation programme. In 2007 they were tested in three test beams: prior to irradiation, after irradiation to $1.010^{14} \mathrm{p} / \mathrm{cm}^{2}$ and after additional irradiation to $1.410^{14} \mathrm{p} / \mathrm{cm}^{2}$ (bringing the cumulative fluence to $2.410^{14} \mathrm{p} / \mathrm{cm}^{2}$ ). The next irradiation will increase the total fluence to $10^{15} \mathrm{p} / \mathrm{cm}^{2}$. There is no indication of decrease in performance between the last two steps, while after the first step there is a slight decrease in signal. The analysis is still ongoing.

\subsection{BCM system performance evaluation}

In 2006 and 2007 in various tests at CERN SPS high energy pion beam (pion momenta ranging from $120-180 \mathrm{GeV} / \mathrm{c}$ ) tests were performed on spare modules and further stages of BCM system (TOT electronics board and back-end FPGA system). For the pion position information a simpler setup of four thin crossed scintillators was used two upstream and two downstream of the tested BCM modules. The readout was triggered on coincidences of all four. Their cross section defined a spot of about $2 \times 2 \mathrm{~mm}^{2}$. The BCM modules were installed on remotely controllable movable $\mathrm{x}$ and $\mathrm{y}$ stages allowing for the whole active surface of the sensors being scanned over the spot defined by scintillators. First analogue signals from BCM front end modules were amplified by Ortec FTA810 fast timing amplifier and recorded by sampling VME ADC module (CAEN V1729, 2GS/s, 12bit ADC). Figure 8 shows a typical signal recorded in ADC counts along with distribution of signals amplitudes for the pions going through central part of the diamond sensor. The probability that signal is larger than 150 ADC counts is shown in Figure 9 as a function of the beam spot position showing uniforme response over the active area of pCVD diamond sensors.
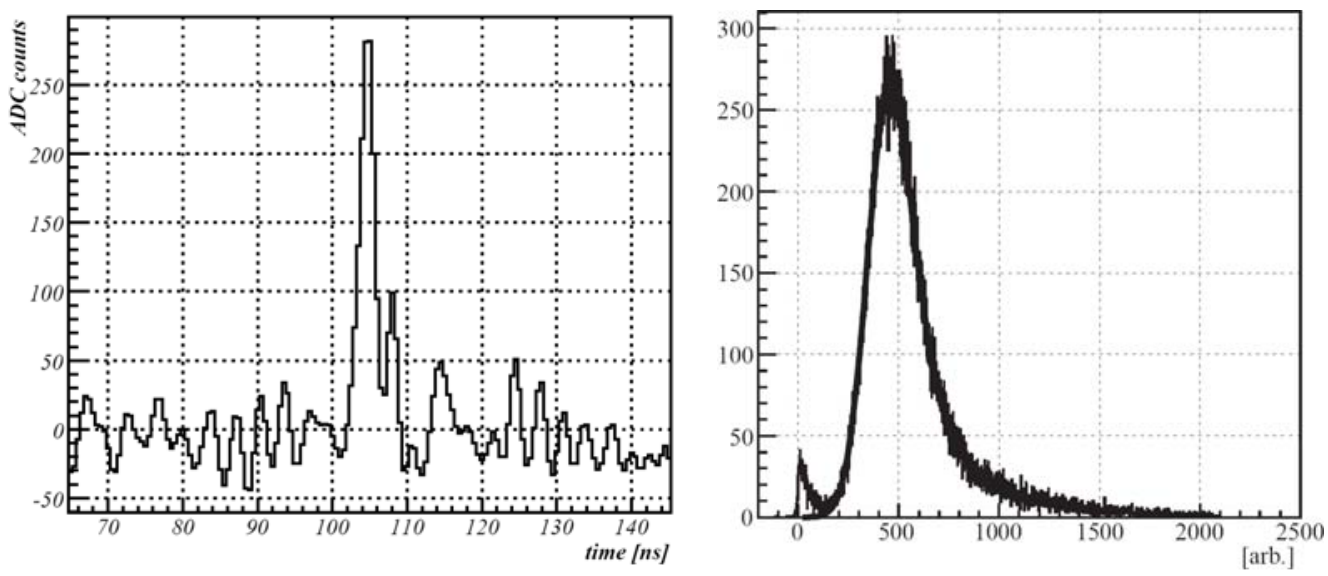

Figure 8: Typical output of front end module recorded in ADC for CERN SPS beam test (left). Signal amplitude distribution is shown on the right plot. 


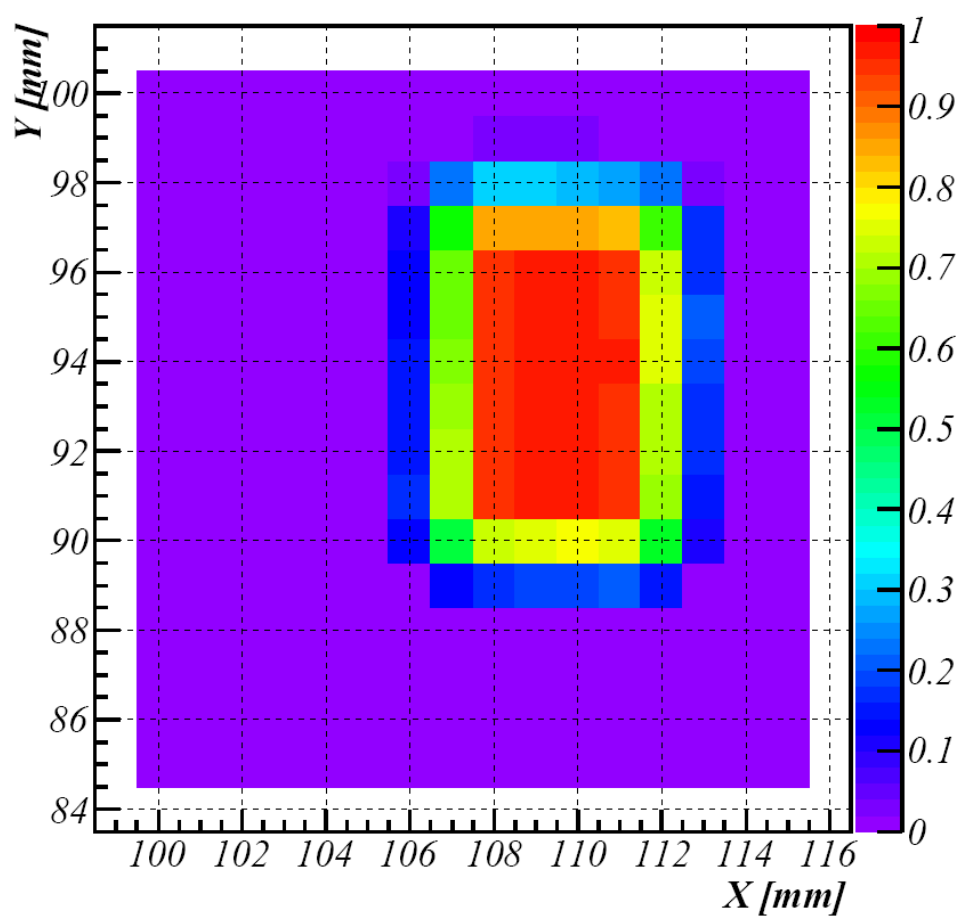

Figure 9: $2 \mathrm{D}$ scan of efficiency of analogue signals $\mathrm{ADC}>150$. The efficiency in the region of diamond sensors is practically $100 \%$. The spot size is $\sim 2 \times 2 \mathrm{~mm}^{2}$, defined by scintillator triggers.

Signals from modules were then taken directly to NINO TOT electronics board and through optical fibres to the opto-receiver board where the NIM monitor output signals were recorded by the same sampling VME ADC module for later analysis. Figure 10 shows efficiency that pion going through the centre of the diamond sensors produce a signal in the output of NINO TOT board for BCM FE module number F406 as a function of NINO discriminator threshold. In absence of pion beam (during short shutdowns) the rate of accidental noise hits was measured at the output of NINO TOT board as a function of the NINO discriminator threshold. Figure 11 shows noise rate in logarithmic scale against the square of threshold applied. The straight line indicates that noise has a Gaussian shape. The two measurements can be combined in a plot of efficiency as a function of the noise rate. Even more indicative is plot of efficiency versus noise occupancy, where noise rate is scaled to $1 \mathrm{LHC}$ bunch to bunch interval of $25 \mathrm{~ns}$. Figure 12 also shows quite a comfortable range of threshold values $(\sim 100 \mathrm{mV}-\sim 170 \mathrm{mV}$ at given settings) at which the BCM system can be operated with fake rate $<10^{-3}$ per bunch crossing and efficiency larger than $99 \%$. The exact level of tolerable fake rate will be determined by simulations and tuned in the final system in ATLAS and will also depend on what kind of logical combination of signals will be used (coincidence noise rate is much smaller than single channel one). Timing of the signals after the full readout chain exercised at CERN SPS beam test (BCM module $\rightarrow$ NINO TOT signal $\rightarrow$ opto-receiver board $\rightarrow$ NIM monitoring output) indicates that the timing resolution of a single channel is about 500 ps (Figure 13). 


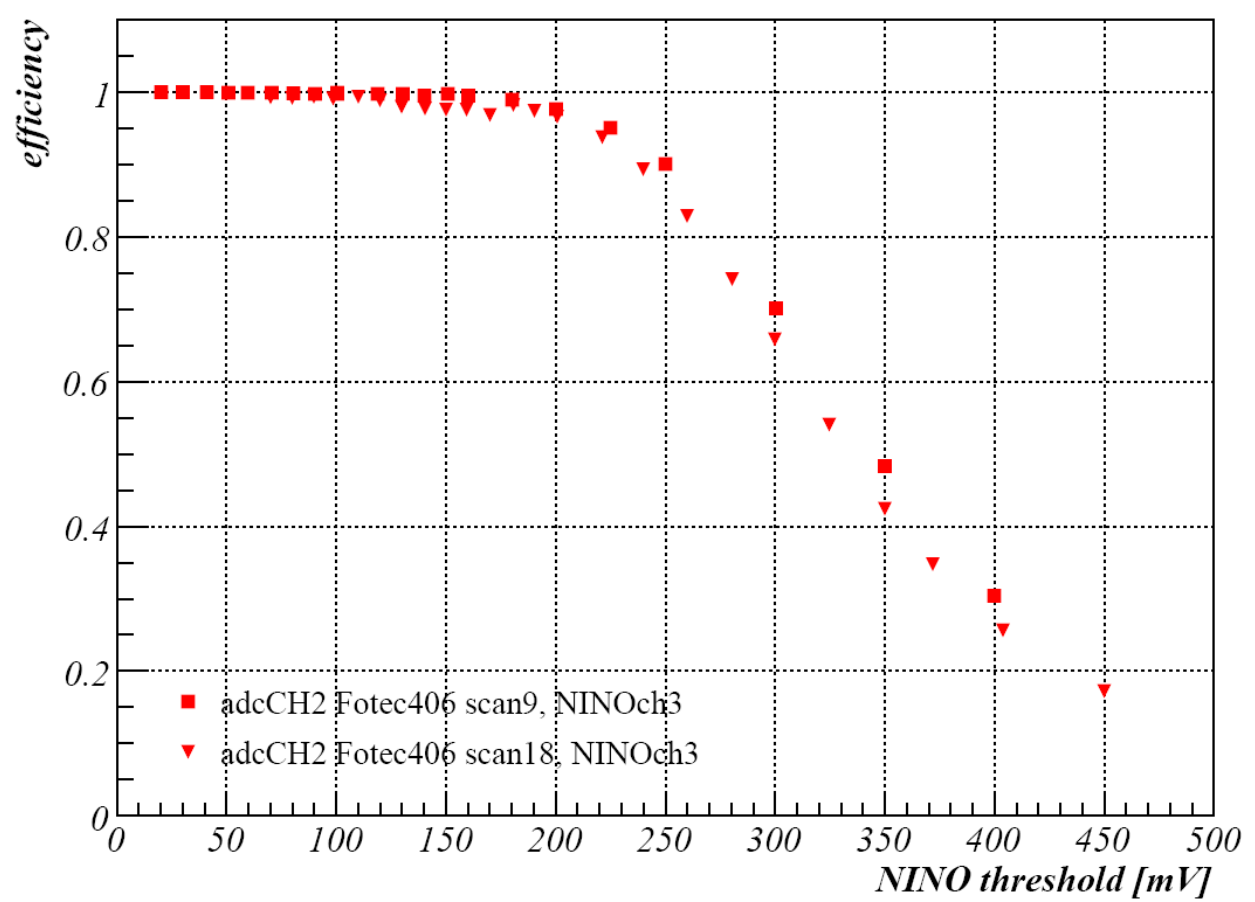

Figure 10: Efficiency of detecting a signal caused by high energy pion impinging on the centre part of diamond sensors installed in BCM module number F406 as function of NINO threshold.

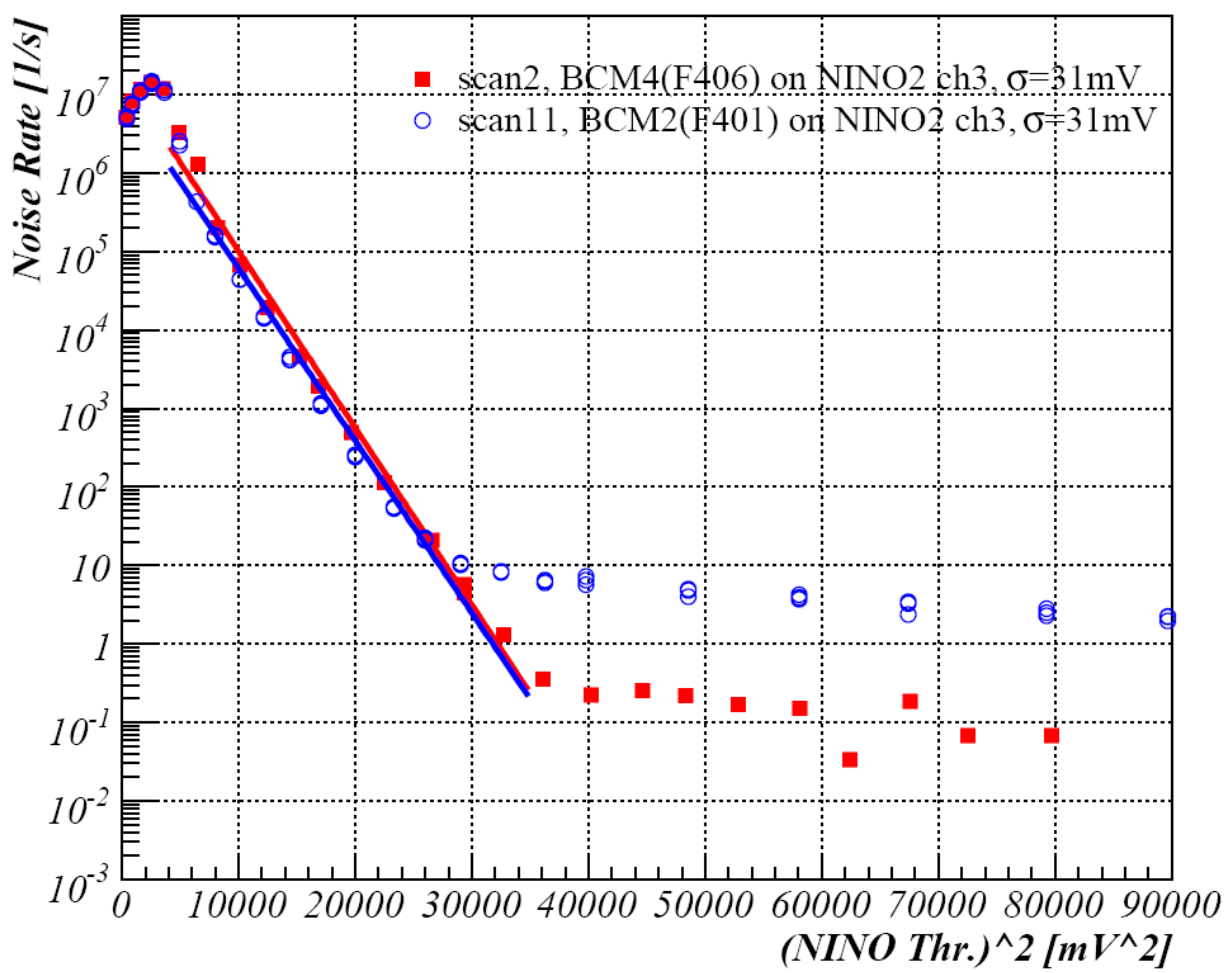

Figure 11: Noise rate measured in the absence of the beam at the output of the optical receiver board versus NINO threshold squared. The plot is compatible with noise of Gaussian shape. 


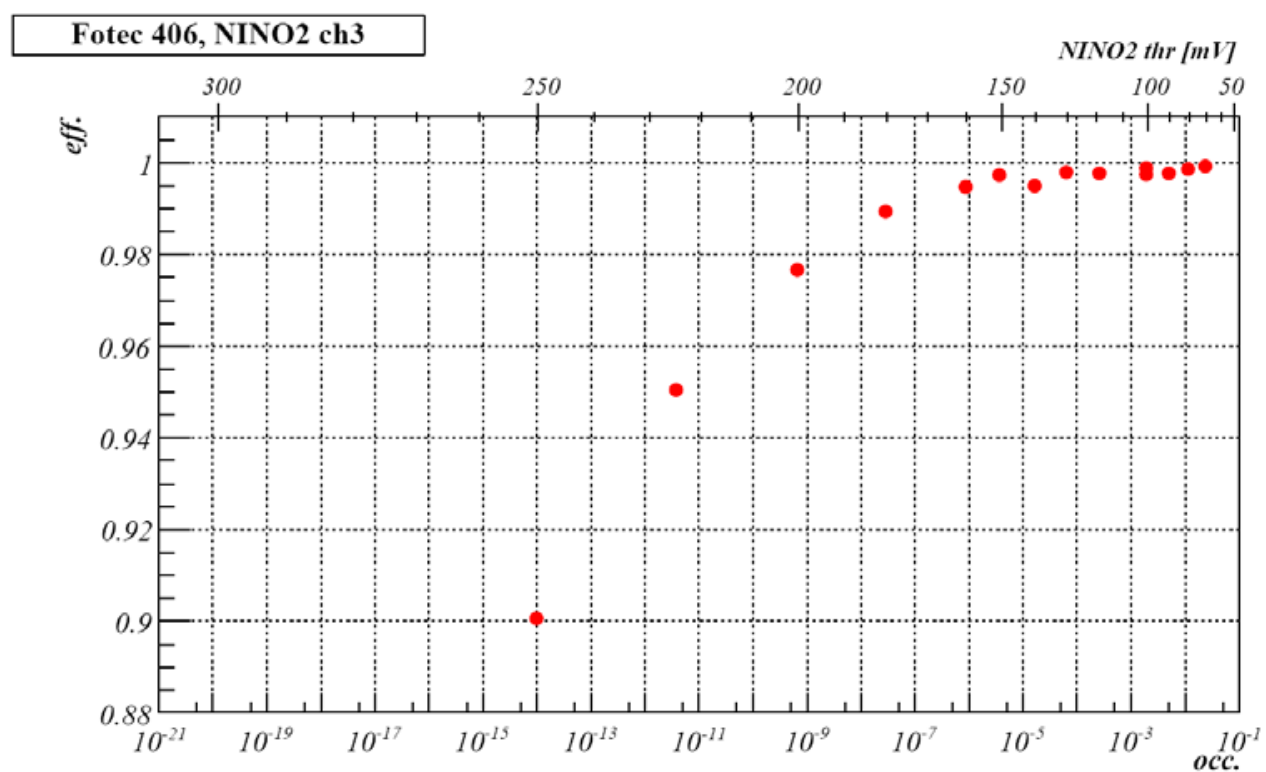

Figure 12: The plot shows the efficiency vs. noise occupancy of BCM system. The noise occupancy is represents the noise rate normalised to $25 \mathrm{~ns}$, the interval between two consecutive LHC bunches. Noise occupancy below $\sim 10^{-7}$ is obtained from the Gaussian fit to the noise rate plot.

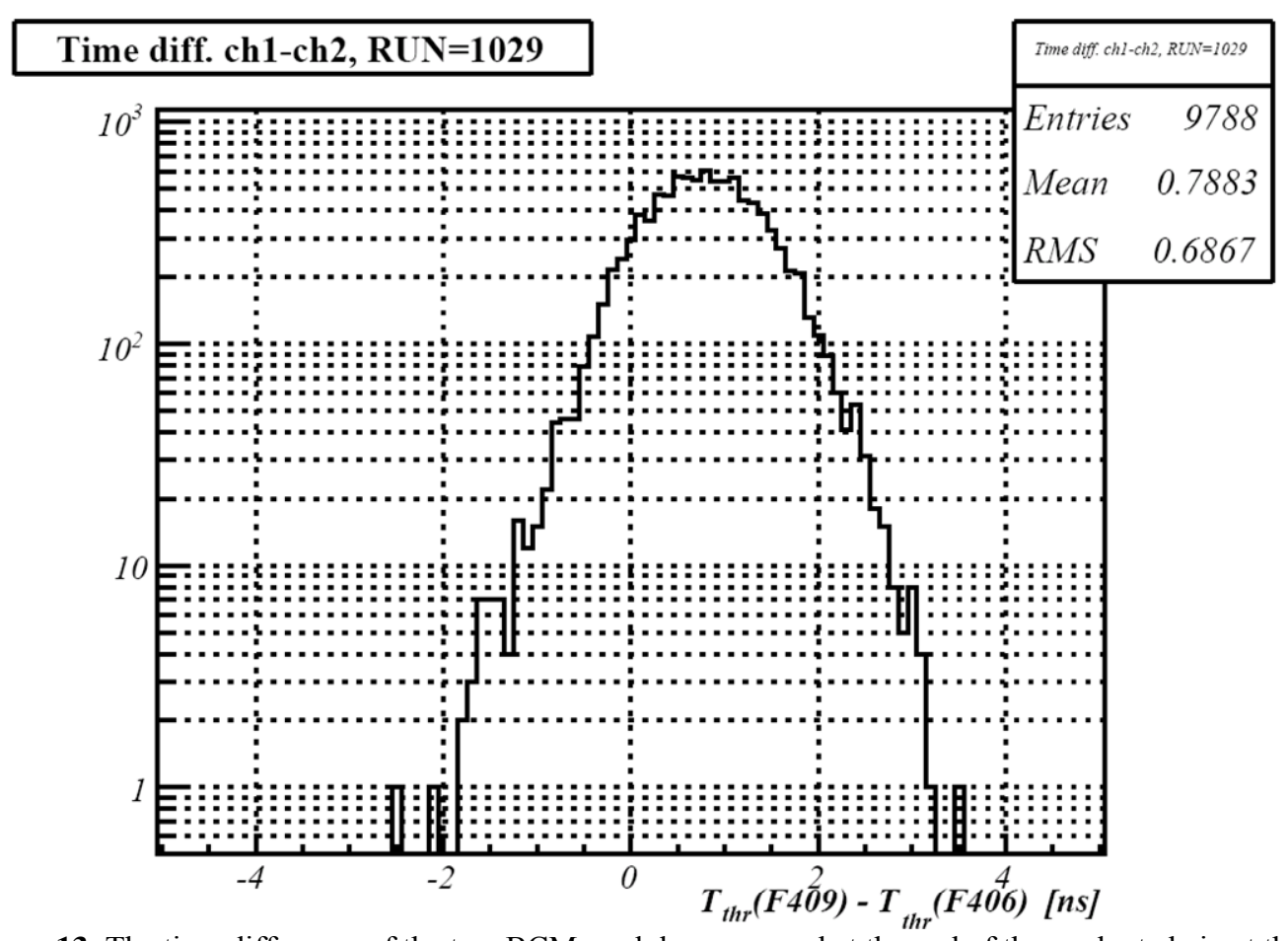

Figure 13: The time difference of the two BCM modules measured at the end of the readout chain at the output of the opto-receiver board. A single module timing resolution is approximately $500 \mathrm{ps.}$ 


\section{Summary}

Recent tests including development of front end design, quality assurance of production modules, evaluation of performance of time-over-threshold NINO readout board and tests of irradiated front end BCM module were presented. The 8 best BCM modules were installed in ATLAS on the Pixel subsystem support structure. All installed modules feature adequate signal to noise figure that averages to about 11:1 for nominal incidences at $45^{\circ}$. It was shown that there is a comfortable interval of suitable threshold levels at which system could be operated keeping the efficiency almost $100 \%$ and noise occupancy low. The timing resolution after full channel readout (up to input to the back end) was shown to be about $500 \mathrm{ps}$.

The focus is now shifting to development of the algorithms for beam conditions and their implementation on the FPGA board. Also missing parts of the hardware and software are being developed that will allow sending BCM information to ATLAS DAQ.

\section{References}

[1] M. Mikuz et al., Diamond pad detector telescope for beam conditions and luminosity monitoring in ATLAS, Nucl. Instr. and Meth. A 579 (2007) 788-794

[2] H. Pernegger, First test results of a high-speed Beam Conditions Monitor for the ATLAS Experiment, IEEE Trans. Nucl. Sci. NS-52 (2005) 1590

[3] H. Frais-Klbl, E. Griesmayer, H. Kagan, H. Pernegger, A Fast Low-Noise Charged-Particle CVD Diamond Detector, IEEE Trans. Nucl. Sci. NS-51 (2004) 3833

[4] F. Anghinolfi, et al., NINO: an ultra-fast and low-power front-end amplifier and discriminator ASCI for the multi-gap resistive plate chambers, Nucl. Instr. and Meth. A 533 (2004) 183-187

[5] CERN RD-42 Collaboration: CVD Diamond Radiation Detector Development (http://rd42.web.cern.ch/RD42/)

[6] Element Six Ltd., Kings Ride Park, Ascot, Berkshire SL5 8BP, UK

[7] A. Gorisek, et al., Atlas diamond Beam Conditions Monitor, Nucl. Instr. and Meth. A 572 (2007) 67-69 